\title{
Biodiesel/Water/Glycerol Emulsion Separation using \\ Electrocoalescence: Model and Process Emulsions
}

Kelly Cristina Ribeiro ${ }^{1}$, Khalil Kashefi ${ }^{1}$, Troner Assenheimer ${ }^{1}$, Cristine Carretoni ${ }^{1}$, Márcio Portilho $^{3}$, José Carlos Pinto ${ }^{2}$, Márcio Nele* ${ }^{*}, 2$

${ }^{1}$ Escola de Química, Universidade Federal do Rio de Janeiro, Cidade Universitária, CEP 21949-900, Rio de Janeiro, RJ, Brazil

2Programa de Engenharia Química, COPPE, Universidade Federal do Rio de Janeiro, CEP 21945-970, Rio de Janeiro, RJ, Brazil

${ }^{3}$ Petrobras/CENPES, Cidade Universitária Q.7, CEP 21949-900 Rio de Janeiro, RJ, Brazil email: nele@eq.ufrj.br

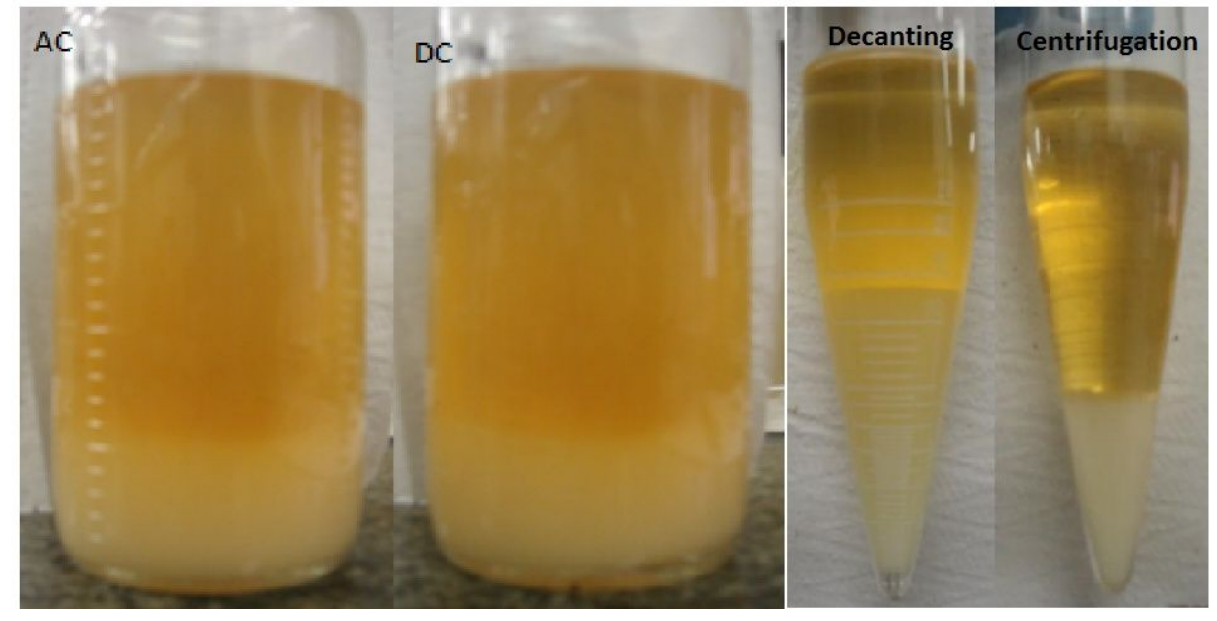

Figure S1. Electrostatic destabilization (using AC and DC fields), decanting, and centrifugation of the products of $R_{2}$ (condition 8). Thick layers of the unresolved emulsion are shown. 


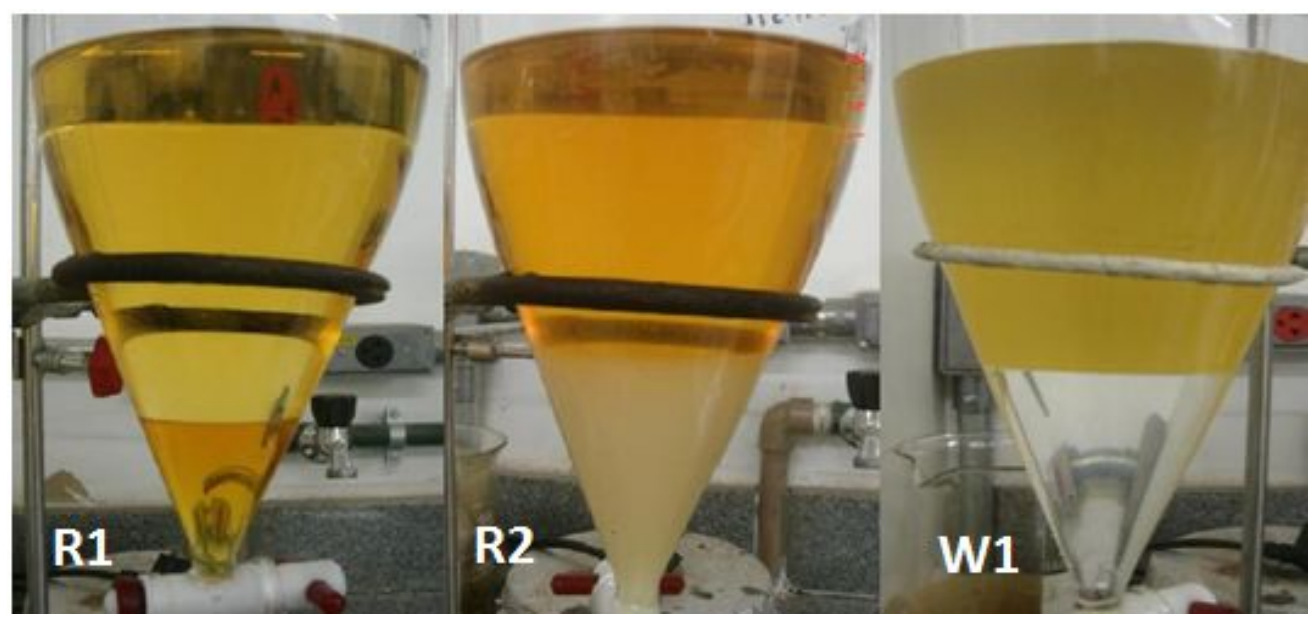

Figure S2. Separation of light and dense phases by decantation in different castor oil biodiesel production steps in the laboratory (condition 15). It shows the process steps $R_{1}, R_{2}$ and $W_{1}$ from left.

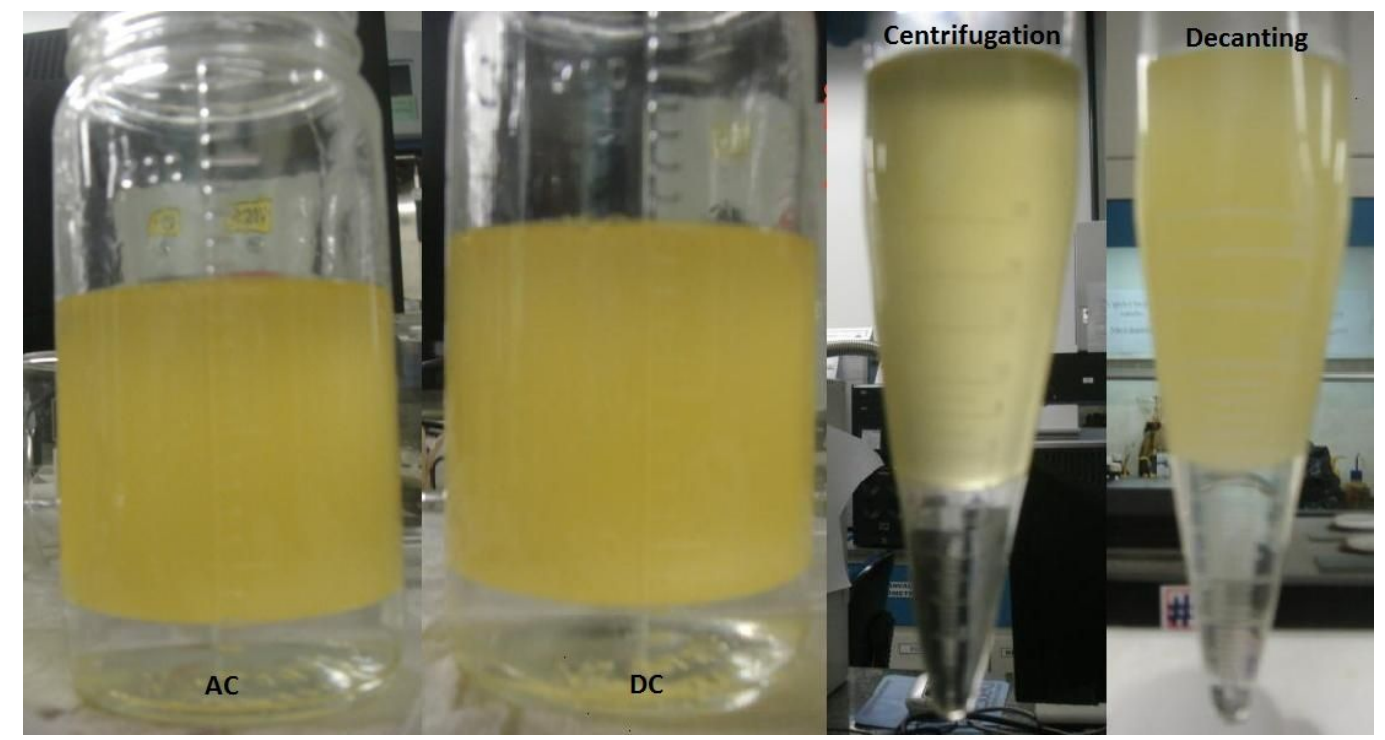

Figure S3. Destabilization of the products of the second washing step $\left(\mathrm{W}_{2}\right)$ with the application of AC and DC fields, centrifugation and decantation, respectively (condition 15). 